\title{
On Solvable Models in Classical Lattice Systems
}

\author{
M. Fannes` and A. Verbeure \\ Instituut voor Theoretische Fysica, Universiteit Leuven, B-3030 Leuven, Belgium
}

\begin{abstract}
We consider one dimensional classical lattice systems and an increasing sequence $\mathscr{S}_{n}(n=1,2, \ldots)$ of subsets of the state space; $\mathscr{S}_{n}$ takes into account correlations between $n$ successive lattice points.

If the interaction range of the potential is finite, we prove that the equilibrium states defined by the variational principle are elements of $\left\{\mathscr{S}_{n}\right\}_{n<\infty}$. Finally we give a new proof of the fact that all faithful states of $\mathscr{S}_{n}$ are DLRstates for some potential.
\end{abstract}

\section{Introduction}

In statistical mechanics one is faced with the problem of computing the free energy density of a system in equilibrium. The variational principle tells us that this free energy is the minimum of the free energy functional as a function of the states. In practice for realistic systems one has to restrict the variation to a parameterizable subset of the set of states, yielding an upper bound for the true free energy.

Here we are dealing with classical lattice systems. The best known approximation is the so-called mean field one. It corresponds to restricting the variation to the set of product states (states without correlations between the lattice sites). The next approximation would be to take into account the nearest neighbour correlations. In the physics literature this is called the Bethe-Peierls method [1-3].

For one dimensional lattices we consider increasing subsets of the set of states: $\mathscr{S}_{1} \subset \mathscr{S}_{2} \subset \ldots \subset \mathscr{S}_{n} \subset \ldots$, where $\mathscr{S}_{n}$ is the set of states taking into account non-trivial correlations between $n$-successive lattice points; $\mathscr{S}_{1}$ is the set of mean field states, $\mathscr{S}_{2}$ is the set of Bethe-Peierls states; the latter is extended to the so-called Bethe lattices. It is clear that better approximations of the free energy are obtained if one considers the variations over the sets $\mathscr{S}_{n}$ with increasing values of $n$.

In this paper we are not concerned with the applications but with the study of the states $\mathscr{S}_{n}$. These states correspond in probability theory to what is called Markov chains with memory of length $n$. We present a new way of defining them

* Bevoegdverklaard navorser NFWO 
without using the notion of conditional expectation. Our definition seems to us to be not only practical for explicit calculation, but might also clarify the BethePeierls and Kikuchi approximation [3] in the physics literature. Our way of looking at it might eventually be extendable to the quantum case [4].

As far as we know, what is new however is our proof that the equilibrium state defined by the variational principle of a system with finite range interactions is necessarily an element of $\mathscr{S}_{n}$ with $n<\infty$.

For completeness we add a new proof of the theorem which can be developed from the works of Dobrushin L. [5], telling that any faithful state of $\mathscr{S}_{n}$ is a DLRstate for some finite range potential. In this proof again, we are not using conditional expectations (as in the description by Markov random fields) but only joint distribution functions.

\section{One Dimensional Models}

Consider the lattice $\mathbb{Z}$; at each site $j \in \mathbb{Z}$ we associate a copy $K_{j}$ of the set $K=\{1, \ldots, N\}$.

For any subset $X \subset \mathbb{Z}$, let $K^{X}=\prod_{j \in X} K_{j}$ be the set of configurations, i.e. the elements of $K^{X}$ are the functions $x_{X}$ from $X$ into $K$; if no confusion is possible we omit the index $X$ of $x_{X}$; if $X \cap Y=\emptyset$, then we denote by $x_{X} \times x_{Y}=x_{X \cup Y}$, the configuration of $X \cup Y$ which coincides on $X$ with $x_{X}$ and on $Y$ with $x_{Y}$; also if $x$ is a configuration of $X^{\prime}$ and $X \subset X^{\prime}$ we denote by $x_{X}$ the restriction of $x$ to $X$.

Let $C_{X}$ be the set of real valued functions on $K^{X} ; C_{X}$ stands for the algebra of observables of the region $X$. Finally the set of continuous functions on $K^{\mathbb{Z}}$ is denoted by $C_{\infty}$. As usual $C_{\infty}$ is the inductive limit of the set $\left\{C_{X} \mid X \subset \mathbb{Z}\right\}$.

Any state $\omega$ of $C_{\infty}$ is a probability measure on $C_{\infty}$, it is described by a family of density distributions, i.e. for any finite volume $X$, there exists a non-negative function $\mu_{X}$ on $K^{X}$ such that:

(i) if $A \in C_{X}$ :

$$
\omega(A)=\sum_{x \in K^{X}} \mu_{X}(x) A(x)
$$

(ii) (normalization)

$$
\sum_{x \in K^{X}} \mu_{X}(x)=1
$$

(iii) (compatibility condition); if $X \subset X^{\prime}$, then

$$
\mu_{X}(x)=\sum_{y \in K^{X^{\prime}-x^{\prime}}} \mu_{X^{\prime}}(x \times y) .
$$

For fixed $n \in \mathbb{N}$, suppose that one has given a family of non-negative functions $\varrho_{i}^{n}$ of the configurations of the interval $[i, i+n-1], i \in \mathbb{Z}$. We suppose that they satisfy

(i) $\sum_{x \in K^{[,, i+n-1]}} \varrho_{i}^{n}(x)=1$, 
(ii) if $\emptyset \neq Y \subset[i, i+n-1] \cap[j, j+n-1]$, let us denote by $Y_{a}^{c}$ the complement of $Y$ in the interval $[a, a+n-1](a=i, j)$, then

(compatibility).

$$
\sum_{x_{Y_{i}^{c}}} \varrho_{i}^{n}\left(x_{Y} \times x_{Y_{i}^{c}}\right)=\sum_{x_{Y_{j}^{c}}} \varrho_{j}^{n}\left(x_{Y} \times x_{Y_{j}}\right)
$$

Finally denote

$$
\varrho_{i}^{n-1}(x)=\sum_{x_{i}+n-1} \varrho_{i}^{n}\left(x \times x_{i+n-1}\right), \quad x \in K^{[i, i+m-2]} .
$$

Our aim is to construct a state $\omega$ for each given family of functions $\varrho_{i}^{n}$. We do this by giving explicitly its density distributions $\mu_{X}$ in terms of the $\varrho_{i}^{n}$.

First we consider the case where $X$ is an interval $[a, b]$ :

(i) if $b-a \geqq n$, then

$$
\begin{aligned}
\mu_{[a, b]}^{n}(x) & =\frac{\prod_{i=a}^{b-n+1} \varrho_{i}^{n}\left(x_{[i, i+n-1]}\right)}{\prod_{i=a}^{b-n} \varrho_{i+1}^{n-1}\left(x_{[i+1, i+n-1]}\right)}, \text { if } \varrho_{i}^{n-1}(\ldots) \neq 0 \\
= & 0 \text { if some } \varrho_{i}^{n-1}(\ldots) \text { vanishes }
\end{aligned}
$$

(ii) if $b-a \leqq n-1$

$$
\mu_{[a, b]}^{n}(x)=\sum_{x_{[b+1, a+n-1]}} \varrho_{a}^{n}\left(x \times x_{[b+1, a+n-1]}\right) .
$$

Now in general let $X=\left\{i_{1}, \ldots, i_{k}\right\} ; i_{1}<\ldots<i_{k}$, then

$$
\mu_{X}^{n}(x)=\sum_{x^{\prime} \in K^{\left[i_{1}, i_{k}\right]-X}} \mu_{\left[i_{1}, i_{k}\right]}^{n}\left(x \times x^{\prime}\right) .
$$

Theorem II.1. The family of functions $\left(\mu_{X}^{n}\right)_{X \subset \mathbb{Z}}$ defined by (5)-(7) are a family of density distributions and hence by (1) define a state $\omega_{n}$ of $C_{\infty}$.

That $\left(\mu_{X X{ }_{X}^{n}}^{n}\right)_{\mathbb{Z}}$ is a family of density distributions follows from the various conditions on the $\varrho_{i}^{n}$ and from the definition of the $\mu_{X}^{n}$. The compatibility condition for the $\mu_{X}^{n}$ follows from conditions (3) and the definitions (4)-(7). The normalization is then a consequence of (4).

Denote by $\mathscr{S}_{n}$ the set of states determined in Theorem II.1. Remark that $\mathscr{S}_{1}$ is the set of product states and that $\mathscr{S}_{1} \subset \mathscr{S}_{2} \subset \ldots \subset \mathscr{S}_{n} \subset \ldots$. Therefore we call this type of states quasi-product states. In probability the corresponding measures arise in the study of Markov chains with memory of length $n$ [4].

Denote by $\tau$ the homomorphism of $\mathbb{Z}$ into the ${ }^{\star}$-automorphisms of the algebra of observables $C_{\infty}$ describing the space translations: $\left(\tilde{\tau}_{a} f\right)(x)=f\left(\tau_{a} x\right), f \in C_{\infty}$, $x \in K^{\mathbb{Z}}$, where $\left(\tau_{a}^{\chi}\right)(\mathrm{i})=x(i+a), i \in \mathbb{Z}$.

Remark that if $\varrho_{i}^{n} \cdot \tau_{a}=\varrho_{i+a}^{n}$ for all $i, a \in \mathbb{Z}$, then the corresponding state $\omega_{n}$ is translation invariant: i.e. $\omega_{n} \cdot \tilde{\tau}_{a}=\omega_{n} ; a \in \mathbb{Z}$.

Given a translation invariant quasi-product state we are now in a position to calculate its entropy density.

Lemma II.2. If $\omega_{n}$ is a translation invariant quasi-product state of $\mathscr{S}_{n}$, determined by the family $\left\{\varrho_{i}^{n}\right\}_{i}$ of functions, then the entropy density $s\left(\omega_{n}\right)$ is given by

$$
s\left(\omega_{n}\right)=-\sum_{x \in K^{[1, n]}} \varrho_{1}^{n}(x) \log \varrho_{1}^{n}(x)+\sum_{x \in K^{[1, n-1]}} \varrho_{1}^{n-1}(x) \log \varrho_{1}^{n-1}(x) .
$$


Proof. We calculate the mean entropy for the interval $[-m, m]$, and then let $m$ tend to infinity:

$$
\frac{S_{[-m, m]}\left(\omega_{n}\right)}{2 m+1}=-\frac{1}{2 m+1} \sum_{x \in K^{[-m, m]}} \mu_{[-m, m]}^{n}(x) \log \mu_{[-m, m]}^{n}(x) .
$$

Using (5) one gets

$$
\begin{aligned}
\frac{S_{[-m, m]}\left(\omega_{n}\right)}{2 m+1}= & -\frac{1}{2 m+1} \sum_{x \in K^{[-m, m]}} \frac{\prod_{i=-m}^{m-n+1} \varrho_{i}^{n}\left(x_{[i, i+n-1]}\right)}{\prod_{i=-m}^{m-n} \varrho_{i+1}^{n-1}\left(x_{[i+1, i+n-1]}\right)} \\
& \cdot\left\{\begin{array}{l}
m-n+1 \\
\left.\sum_{j=-m}^{m} \log \varrho_{j}^{n}\left(x_{[j, j+n-1]}\right)-\sum_{j=-m}^{m-n} \log \varrho_{j+1}^{n-1}\left(x_{[j+1, j+n-1]}\right)\right\} .
\end{array}\right.
\end{aligned}
$$

From (4):

$$
\begin{aligned}
\frac{S_{[-m, m]}\left(\omega_{n}\right)}{2 m+1}= & -\frac{1}{2 m+1} \sum_{j=-m}^{m-n+1} \sum_{x \in K^{[j, j+n-1]}} \varrho_{j}^{n}(x) \log \varrho_{j}^{n}(x) \\
& +\frac{1}{2 m+1} \sum_{j=-m}^{m-n} \sum_{x \in K^{[j+1, j+n-1]}} \varrho_{j+1}^{n-1}(x) \log \varrho_{j+1}^{n-1}(x) .
\end{aligned}
$$

Finally as $\omega_{n}$ is translation invariant $\varrho_{j}^{n}=\varrho_{1}^{n} \cdot \tau_{j-1}$, one finds:

$$
\begin{aligned}
\frac{S_{[-m, m]}\left(\omega_{n}\right)}{2 m+1}= & -\frac{2 m-n+2}{2 m+1} \sum_{x \in K^{[1, n]}} \varrho_{1}^{n}(x) \log \varrho_{1}^{n}(x) \\
& +\frac{2 m-n+1}{2 m+1} \sum_{x \in K^{[1, n-1]}} \varrho_{1}^{n-1}(x) \log \varrho_{1}^{n-1}(x)
\end{aligned}
$$

and with $m$ tending to infinity one gets the result.

Now take any state $\omega$ of $C_{\infty}$ with a family of density distributions $\left\{\mu_{X}\right\}_{X \in \mathbb{Z}}$. For a fixed given $n \in \mathbb{N}$, we associate to $\omega$ a quasi-product state $\omega_{n}$ in the following way.

Define the family of non-negative functions $\varrho_{i}^{n}$ by $\varrho_{i}^{n}(x)=\mu_{[i, i+n-1]}(x)$. It is clear that they satisfy the properties (4) and (5). The quasi-product state $\omega_{n}$ is defined by these functions $\varrho_{i}^{n}(x)$ as in Theorem II.1.

Lemma II.3. Let $[a, b]$ be any interval of the lattice. Then

$$
S_{[a, b]}(\omega) \leqq S_{[a, b]}\left(\omega_{n}\right),
$$

i.e.the entropy of the state $\omega$ is majorized by the entropy of its associated quasiproduct state.

Proof. If $b-a<n$ then clearly $S_{[a, b]}(\omega)=S_{[a, b]}\left(\omega_{n}\right)$. Suppose now $b-a \geqq n$, then

$$
S_{[a, b]}(\omega)-S_{[a, b]}\left(\omega_{n}\right)=\sum_{x \in K^{[a, b]}}\left\{\mu_{[a, b]}^{n}(x) \log \mu_{[a, b]}^{n}(x)-\mu_{[a, b]}(x) \log \mu_{[a, b]}(x)\right\} .
$$

Using the convexity of $x \in[0,1] \rightarrow x \log x \in \mathbb{R}$ :

$$
x_{0} \log x_{0}-x \log x \leqq\left(x_{0}-x\right)\left(1+\log x_{0}\right),
$$


one gets

$$
\begin{aligned}
S_{[a, b]}(\omega)-S_{[a, b]}\left(\omega_{n}\right) & \leqq \sum_{x \in K^{[a, b]}}\left(\mu_{[a, b]}^{n}(x)-\mu_{[a, b]}(x)\right)\left(1+\log \mu_{[a, b]}^{n}(x)\right) \\
& =\sum_{x \in K^{[a, b]}}\left(\mu_{[a, b]}^{n}(x)-\mu_{[a, b]}(x)\right) \log \mu_{[a, b]}^{n}(x),
\end{aligned}
$$

which vanishes because $\log \mu_{[a, b]}^{n}(x)$ is a sum of terms depending only on the configurations of intervals of length equal to $n$ or $n-1$. On these intervals $\mu=\mu^{n}$.

Corollary II.4. With the above notations, if $\omega$ is a translation invariant state, then also the corresponding quasi-product state $\omega_{n}$ is translation invariant, and the entropy densities satisfy $s(\omega) \leqq s\left(\omega_{n}\right)$.

As usual let $\phi$ be a translation invariant potential of range $n$, i.e. a map from the finite subsets $X$ of $\mathbb{Z}$ in $\phi(X) \in C_{X}$ such that $\phi(X)=0$ if $X$ is not contained in a interval of length less than or eqal to $n$.

The corresponding local Hamiltonians are then, for $\Lambda \subset \mathbb{Z}$ :

$$
H_{\Lambda}=\sum_{X \subset A} \phi(X) \text {. }
$$

Let $\omega$ be any translation invariant state. Then its free energy density is defined to be $f(\omega)=e(\omega)-\beta s(\omega)$, where

$$
e(\omega)=\lim _{|\Lambda| \rightarrow \infty} \frac{\omega\left(H_{\Lambda}\right)}{|\Lambda|}, \quad|\Lambda|=\text { volume of } \Lambda, \text { and } \beta>0 .
$$

Now the state $\omega^{\beta}$ is an equilibrium state of the system at inverse temperature $\beta$, if it minimizes the free energy density.

Theorem II.5. If $\phi$ is a translation invariant potential of range $n$ then the equilibrium state is a quasi-product state, element of $\mathscr{S}_{n}$.

Proof. One computes

$$
e(\omega)=\sum_{0 \in X} \frac{\omega(\phi(X))}{|X|} .
$$

As $\phi$ has a range equal to $n, e(\omega)=e\left(\omega_{n}\right)$, where $\omega_{n}$ is the quasi-product state associated to $\omega$. Hence, using Corollary II.4: $f(\omega)=e(\omega)-\beta s(\omega)$ $\geqq e\left(\omega_{n}\right)-\beta s\left(\omega_{n}\right)=f\left(\omega_{n}\right)$. Therefore

$$
\inf _{\omega} f(\omega)=\inf _{\omega_{n} \in \mathscr{S}_{n}} f\left(\omega_{n}\right),
$$

and the existence of a solution in $\mathscr{S}_{n}$ is guaranteed by the usual arguments of semicontinuity of the map $\omega \rightarrow f(\omega)$. It is known that the solution is unique [6].

Remark that the variational problem characterizing the equilibrium state is a problem with a finite number of variables $\left(\varrho^{n}(x)\right)$. For this reason it is called a soluble model. Moreover by [5-7] and Theorem II.1 we know the quasi-product structure of the equilibrium state, i.e. we know not only the thermodynamics but all correlations. At this point one might refer to an earlier reference $[7,8]$. In [7] the equilibrium state is studied by the transfer matrix method and compared with the variational approach. In [8] explicit equations for the correlation functions are studied for the nearest and next nearest neighbour Ising chains. 
Next one can ask for the inverse question: namely, given any faithful state $\omega_{n} \in \mathscr{S}_{n}$, is that state an equilibrium state of a certain model? The answer to this question is known (e.g. [5]). However we want to add it not only for completeness but also we want to take this opportunity to write down our form of DLR-equations which evidently holds for any dimension.

Let $Q$ be the group of local bijections of $K^{\mathbb{Z}}$ into itself: i.e. $U \in Q$ if there exists a finite $\Lambda \subset \mathbb{Z}$ such that: $(U x)(i)=x(i)$ for all $i \in \Lambda^{c}, x \in K^{\mathbb{Z}}$. The smallest set $\Lambda$ with this property is called the support of $U$. Furthermore, denote by $\tilde{U}$ the transposed of $U \in Q$ to $C_{\infty}$, i.e. $(\tilde{U} f)(x)=f\left(U^{-1} x\right), f \in C_{\infty}, x \in K^{\mathbb{Z}}$.

Definition II.6. A map $\Delta$ from $Q$ into $C_{\infty}$ is called a relative Hamiltonian if for any pair $U_{1}, U_{2} \in Q$ holds: $\Delta_{U_{1} U_{2}}=\tilde{U}_{1} \Delta_{U_{2}}+\Delta_{U_{1}}$.

Proposition II.7. For a given relative Hamiltonian $\Delta$ there exists a family of local Hamiltonians $H_{\Lambda} \in C_{\Lambda}$ such that for $W \in Q: \Delta_{W}=\lim _{\Lambda} W H_{\Lambda}-H_{\Lambda}$.

Proof. Fix $x \in K^{\mathbb{Z}}$. For any $\Lambda \subset \mathbb{Z}$ we construct $H_{\Lambda}$. Take $y \in K^{\mathbb{Z}}$ and $\Lambda \subset \mathbb{Z}$; denote by $Q_{\Lambda}$ all transformations with support in $\Lambda$; one finds at least one $U \in Q_{\Lambda}$. such that $x_{\Lambda}=U y_{\Lambda}$.

Define $H_{\Lambda}(y)=\Delta_{U}\left(x_{\Lambda}\right) . H_{\Lambda}$ is well defined. Indeed, take any other map $V \in Q_{\Lambda}$ such that $x_{A}=V y_{A}$ then we prove that $\Delta_{U}(x)=\Delta_{V}(x)$. We have

$$
\begin{aligned}
\Delta_{U V^{-1}}(x) & =U \Delta_{V^{-1}}(x)+\Delta_{U}(x) \\
& =\Delta_{V^{-1}}\left(y_{\Lambda} \times x_{\Lambda^{c}}\right)+\Delta_{U}(x) \\
& =\Delta_{V^{-1}}\left(V^{-1} x_{\Lambda} \times x_{\Lambda^{c}}\right)+\Delta_{U}(x) \\
& =V \Delta_{V^{-1}}(x)+\Delta_{U}(x) .
\end{aligned}
$$

But as $0=\Delta_{V V^{-1}}(x)=V \Delta_{V^{-1}}(x)+\Delta_{V}(x)$, one gets $\Delta_{U V^{-1}}(x)=-\Delta_{V}(x)+\Delta_{U}(x)$. Hence, it remains to prove that $\Delta_{U V^{-1}}(x)=0$.

As $Q_{A}$ is a finite group, there exists a positive number $n$ such that $K^{n}$ equals the identity with $K=U V^{-1} \in Q_{\Lambda}$. Also $K x=K^{-1} x=x$. Therefore

$$
0=\Delta_{K^{n}}(x)=K \Delta_{K^{n-1}}(x)+\Delta_{K}(x)=\Delta_{K^{n-1}}(x)+\Delta_{K}(x) .
$$

By recurrence $0=n \Delta_{K}(x)$ or $\Delta_{U V^{-1}}(x)=0$. Finally take any $W \subset Q$, then

$$
\begin{gathered}
\lim _{\Lambda}\left(W H_{\Lambda}(y)-H_{\Lambda}(y)\right)=\lim _{\Lambda}\left(H_{\Lambda}\left(W^{-1} y\right)-H_{\Lambda}(y)\right)=\lim _{\Lambda}\left(\Delta_{U W}(x)-\Delta_{U}(x)\right) \\
\quad=\lim _{\Lambda} U \Delta_{W}(x)=\lim _{\Lambda} \Delta_{W}\left(U^{-1} x_{\Lambda} \times x_{\Lambda^{c}}\right)=\lim _{\Lambda} \Delta_{W}\left(y_{\Lambda} \times x_{\Lambda^{c}}\right)=\Delta_{W}(y) .
\end{gathered}
$$

Now we define the equilibrium states by means of the DLR-conditions avoiding a formulation in terms of conditional probabilities [9].

Definition II.8. A state $\omega$ of $C_{\infty}$ satisfies the DLR-conditions at inverse temperature $\beta=1$ for a relative Hamiltonian $\Delta$ if for all $f \in C_{\infty}$ and $U \in Q: \omega\left(\tilde{U}^{-1} f\right)$ $=\omega\left(f \exp \left(-\Delta_{U}\right)\right)$.

Denote by $Q_{0}$ the subset of $Q$ containing only the "one-point" transformations, i.e. those acting only on one lattice site. Next we prove a proposition covering the result of Dobrushin [10] which says that a state is a DLR-state if and only if all its onepoint conditional probabilities are given by Gibbs factors. 
Proposition II.9. A state $\omega$ of $C_{\infty}$ satisfies the DLR-conditions at $\beta=1$ for a relative Hamiltonian $\Delta$ if for all $f \in C_{\infty}$ and $U \in Q_{0}$ holds

$$
\omega\left(\tilde{U}^{-1} f\right)=\omega\left(f \exp \left(-\Delta_{U}\right)\right) .
$$

Proof. Remark that any configuration of a finite volume can be transformed in any other by a finite product of elements of $Q_{0}$. Because of the presence in $C_{\infty}$ of the projections on any fixed local configuration it is sufficient to have the DLR-equations for all $U$ 's which are finite products of one-point transformations.

Finally if one has the DLR-equations for all one-point transformations, one has it for all products of one-point transformations. Indeed, suppose $U_{1}, U_{2} \in Q_{0}$, then

$$
\begin{aligned}
\omega\left(\tilde{U}_{1} \tilde{U}_{2}(f) \exp \left(-\Delta_{U_{1} U_{2}}\right)\right) & =\omega\left(\tilde{U}_{1}\left(\tilde{U}_{2} f\right) \exp \left(-U_{1} \Delta_{U_{2}}-\Delta_{U_{1}}\right)\right) \\
& =\omega\left(\tilde{U}_{1}\left(\tilde{U}_{2}(f) \exp -\Delta_{U_{2}}\right) \exp -\Delta_{U_{1}}\right) \\
& =\omega\left(\tilde{U}_{2}(f) \exp \left(-\Delta_{U_{2}}\right)\right) \\
& =\omega(f)
\end{aligned}
$$

and by recurrence for all finite products of $U$ 's.

After this presentation of the DLR-equations we come back to the study of our quasi-product states.

Theorem II.10. Let $\omega_{n} \in \mathscr{S}_{n}$ be faithful and $U \in Q$ with support $\Lambda$. Denote by $\tilde{\Lambda}$ the interval

$$
\left[\min _{i \in \Lambda}(i-n+1), \max _{i \in \Lambda}(i+n-1)\right] .
$$

Then $\omega_{n}$ satisfies the DLR-conditions (II.8) with relative Hamiltonian

$$
\Delta_{U}(x)=\log \frac{\mu_{\tilde{\Lambda}}^{n}\left(x_{\tilde{\Lambda}}\right)}{\mu_{\tilde{\Lambda}}^{n}\left(U^{-1} x_{\tilde{\Lambda}}\right)}, \quad x \in K^{\mathbb{Z}} .
$$

Proof. As $\omega_{n}$ is faithful, it is clear that $\Delta_{U}$ as defined by (8) belongs to $C_{\widetilde{\Lambda}}$ and satisfies the relations of (II.6). Now take $f \in C_{\Lambda}$, where $\Lambda^{\prime}$ is any interval containing $\tilde{\Lambda}$, then

$$
\omega_{n}\left(f e^{-\Lambda_{U}}\right)=\sum_{x \in K^{\Lambda^{\prime}}} f(x) \mu_{\Lambda^{\prime}}^{n}(x) \frac{\mu_{\Lambda}^{n}\left(U^{-1} x\right)}{\mu_{\Lambda}^{n}(x)} .
$$

As support of $U$ equals $\Lambda$, use formula (7) to get

$$
\omega_{n}\left(f e^{-\Delta_{U}}\right)=\sum_{x \in K^{\Lambda^{\prime}}} f(x) \mu_{\Lambda^{\prime}}^{n}\left(U^{-1} x\right)=\omega_{n}\left(\tilde{U}^{-1} f\right) .
$$

By Theorem II.10 we showed that any faithful state of the type described in Theorem II.1 does satisfy the DLR-equations and that the relative Hamiltonian can be computed in terms of the defining function of the state.

Normally a physical system is given in terms of an interaction potential or in terms of local Hamiltonians. Next we solve the question whether one can find such a potential in terms of a given family of functions $\left(\varrho_{i}^{n}\right)_{i \in \mathbb{Z}}$.

Define

$$
\phi(X)=0
$$


Fig. 1

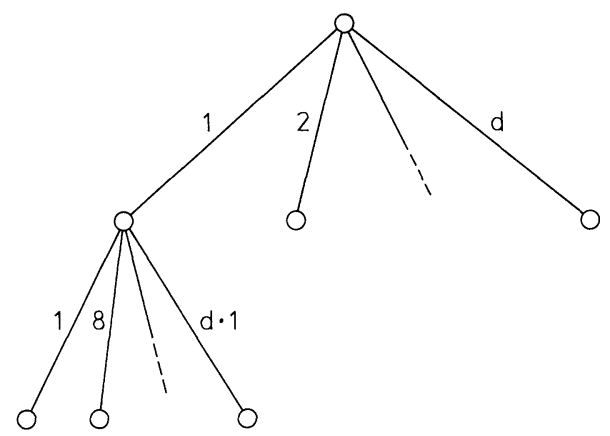

if $X$ is not an interval $[i, i+n-1]$ for some $i \in \mathbb{Z}$, and

$$
\exp \phi([i, i+n-1])(x)=\frac{\varrho_{i}^{n}\left(x_{[i, i+n-1]}\right)}{\varrho_{i}^{n-1}\left(x_{[i, i+n-2]}\right)^{1 / 2} \varrho_{i+1}^{n-1}\left(x_{[i+1, i+n-1]}\right)^{1 / 2}} .
$$

Compute now

$$
\begin{aligned}
\Delta_{U_{k}}(x) & =\lim _{\Lambda}\left(\widetilde{U}_{k} H_{\Lambda}-H_{\Lambda}\right)(x) \\
& =\sum_{l=1}^{n}\left(\phi([k-n+l, k+l-1])\left(U_{k}^{-1} x\right)-\phi([k-n+l, k+l-1])(x)\right) .
\end{aligned}
$$

Then one verifies:

$$
\exp -\Delta_{U_{k}}(x)=\frac{\mu_{\Lambda}^{n}\left(U_{k}^{-1} x\right)}{\mu_{\Lambda}^{n}(x)}
$$

for any $\Lambda$ large enough $\left(\Lambda \supset[k-n+1, k+n-1]\right.$ and where $\mu_{\Lambda}^{n}$ is defined as in (5) and (6).

This proves

Theorem II.11. Any faithful state $\omega_{n} \in \mathscr{S}_{n}$ is a DLR-state for a Hamiltonian constructed from a $n$-body potential defined by (9). The potential is uniquely defined.

\section{Models on a Tree}

Here we consider a lattice tree $B$, this is a connected graph such that each site has $d$ neighbours; two points $i, j \in B$ are neighbours if they are endpoints of a bond, denoted by $\langle i, j\rangle$; the graph does not have closed paths (or cycles), hence two different sites are connected by one and only one path (see figure).

Again the configuration space is $K^{B}$ and the algebra of observables is taken to be $C\left(K^{B}\right)$. Further the notation of Sect. II extends in a natural manner to this kind of lattices in a way that an interval becomes now a connected subgraph.

For any subset $X$ of the lattice denote by $\Gamma(X)$ the smallest connected subgraph containing all the points $x_{1}, \ldots, x_{n}$. We use the notation $n_{i}^{T}$ for the degree of a point $i$ in $\Gamma$, i.e. the number of incident bonds $\langle i, j\rangle$ with $j \in \Gamma$. 
Suppose that for any bond $\langle i, j\rangle$ one is given a non-negative function $\varrho_{\langle i, j\rangle}$ satisfying the following conditions:

(i) $\sum_{x \in K\{i, j\}} \varrho_{\langle i, j\rangle}(x)=1$

(ii) for any pair of bonds $\langle i, j\rangle,\langle i, k\rangle$

$$
\sum_{x \in K^{\{j\}}} \varrho_{\langle i, j\rangle}(y \times x)=\sum_{x \in K^{\{k\}}} \varrho_{\langle i, k\rangle}(y \times x) \equiv \varrho_{\{i\}}(y) \text { for } y \in K^{\{i\}} .
$$

For any such family $\left\{\varrho_{\langle i, j\rangle}\right\}$ of functions we define a family of density distributions $\left\{\mu_{X}^{\varrho}\right\}_{X \subset \boldsymbol{B}}$.

Let $\Gamma$ be a connected subgraph, then

$$
\begin{aligned}
& \mu_{\Gamma}^{\varrho}(x)=\frac{\prod_{\langle i, j\rangle ; i, j \in \Gamma} \varrho_{\langle i, j\rangle}\left(x_{\langle i, j\rangle}\right)}{\prod_{i \in \Gamma} \varrho_{\{i\}}\left(x_{\{i\}}\right)^{n_{i}^{\Gamma}-1}}, \quad \text { if all } \varrho_{\{i\}}>0, \\
& =0 \quad \text { if some } \varrho_{\{i\}}\left(x_{\{i\}}\right)=0 .
\end{aligned}
$$

For any arbitrary subset $X$, define

Then we have

$$
\mu_{X}^{\varrho}(x)=\sum_{y \in K^{\Gamma(X)}-\mathbf{x}} \mu_{\Gamma(X)}^{\varrho}(x \times y) .
$$

Theorem III.1. The family of functions $\left(\mu_{X}^{\varrho}\right)_{X \subset B}$, defined by (10) are a family of density distributions and hence define a state $\omega^{\varrho}$ of $C\left(K^{B}\right)$.

Remark that in the case when the number of neighbours $d$ of the lattice $B$ equals two, then the states described by Theorem II.1 coincide with the class $\mathscr{S}_{2}$ of Theorem II.1. It is not evident how to define on a tree $(d>2)$ the class of states corresponding to the set $\mathscr{S}_{n}$ with $n>2$.

As in Theorem II.10 we can also prove that any faithful state of the type described by Theorem III.1 satisfies the DLR-conditions for a certain relative Hamiltonian. Also as in Theorem II.11 one can find a nearest-neighbour two-body potential associated to this relative Hamiltonian. This class of models contains the usual Ising model, Potts model, etc.

Next one wishes to consider the variational problem to characterize the equilibrium state. To this end one introduces a notion of translation invariance. Let $\tau$ be any bijection of the graph into itself, then the state $\omega^{\varrho}$ is called translation invariant if its defining bond function $\varrho_{\langle i, j\rangle}$ satisfies $\varrho_{\tau\langle i j\rangle}\left(x_{\tau\langle i j\rangle}\right)=\varrho_{\langle i j\rangle}\left(x_{\langle i j\rangle}\right)$ for all bonds $\langle i j\rangle$.

To compute the free energy density functional, one follows the usual procedure of taking the thermodynamic limit of the local free energies computed for a sequence of volumes which is increasing and absorbing. Remark that for a tree, the boundary (set of lattice sites connected to the finite graph by only one bond) is of the same order of magnitude as the total volume.

As for the one-dimensional chain one computes the entropy density

$$
s\left(\omega^{\varrho}\right)=-\sum_{x \in K^{\langle i j\rangle}} \varrho_{\{i j\}}(x) \log \varrho_{\langle i j\rangle}(x)+\sum_{x \in K^{\{i\}}} \varrho_{\{i\}}(x) \log \varrho_{\{i\}}(x) .
$$

For a nearest neighbour interaction this leads to a free energy density functional which is exactly the same as in Sect. I for states in $\mathscr{S}_{2}$. 
For arbitrary higher dimensional lattices the so-called Bethe approximation consists in associating weight factors $\gamma_{1}$ and $\gamma_{2}$ to the contributions of the sites, respectively of the bonds in formula (11). These factors are chosen as a function of the coordination number $\left(n_{i}\right)$ and eventually of other parameters of the lattive. It is unclear except for the one-dimensional lattice, that this yields an upper bound for the free energy of the original system.

\section{References}

1. Bethe, H.A.: On the annihilation radiation of positrons, Proc. R. Soc. A150, 129 (1935)

2. Peierls, R.: Proc. Camb. Philos. Soc. A32, 471 (1936)

3. Hymans, J., de Boer, J.: An approximation method for order-disorder problems I, and II. Physica 21, 471 (1955) and 21, 485 (1955)

4. Accardi, L.: Topics in quantum probability. Phys. Rep. 77, 169 (1981)

5. Spitzer, F.: Am. Math. Monthly 78, 142 (1971)

6. Ruelle, D.: Statistical mechanics of a one-dimensional lattice gas. Commun. Math. Phys. 9, 267 (1968)

7. Brascamp, H.J.: Equilibrium states for a one dimensional lattice gas. Commun. Math. Phys. 21, 56 (1971)

8. Vertogen, G., de Vries, A.S.: The Ising problem. Commun. Math. Phys. 29, 131 (1973)

9. Shiga, T.: Z. Wahrsch. Theor. Verw. Geb. 39, 339 (1977)

10. Dobrushin, R.L.: Theor. Prob. Appl. 13, 197 (1968)

Communicated by H. Araki

Received April 16, 1984; in revised form June 28, 1984 